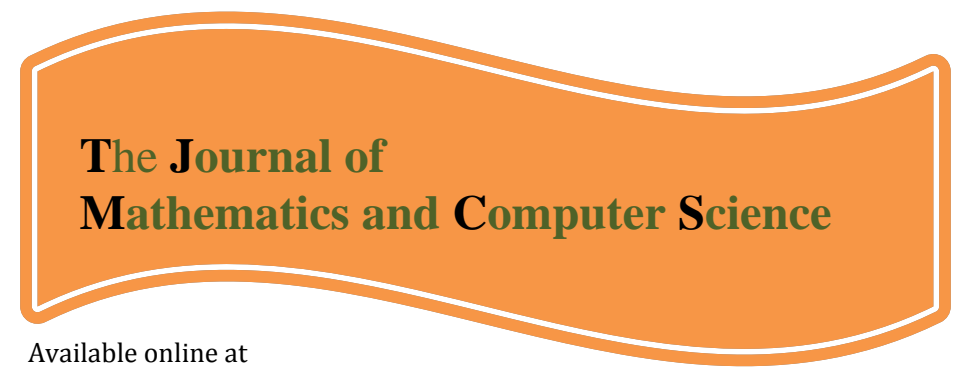

http://www.TJMCS.com

The Journal of Mathematics and Computer Science Vol .2 No.1 (2011) 20-26

\title{
The Progressions of Fuzzy Numbers and Their Features
}

\author{
Ezzatallah Baloui Jamkhaneh ${ }^{1,}{ }^{*}$, Ali Shabani2 ${ }^{2}$, Roghayeh Zareei Jamkhaneh ${ }^{3}$, Mohamad Khaleghi ${ }^{4}$ \\ 1,2,4Department of Statistics, Islamic Azad University, Ghaemshahr Branch, Ghaemshahr, Iran.e_baloui2008@yahoo.com \\ 3Islamic Azad University,Sari Branch,Sari, Iran. vbaloui@yahoo.com
}

Received: July 2010, Revised: October 2010

Online Publication: January 2011

\begin{abstract}
In this paper a certain progression of fuzzy numbers is introduced that called geometrical- arithmetical progression of fuzzy numbers. We study the features of this kind of progression. We also introduced the geometrical progression of fuzzy numbers and the arithmetical progression of fuzzy numbers through using geometrical-arithmetical progression. If fuzzy numbers change into crisp numbers, then geometrical- arithmetical progression of crisp numbers is obtained which is more general than geometrical and arithmetical progression and in special cases, change into them. There are some numerical examples at the end.
\end{abstract}

Keywords: Fuzzy number, arithmetical progression, geometrical progression, geometrical- arithmetical progression.

\section{Introduction}

Progressions are widely used in computing the interest annually of a bank, half-life of radioactive substances, the rate of growth and etc. In conventional geometrical and arithmetical progressions, numbers are certain and in the form of crisp. In conventional geometrical progression, the sentences are successively multiplied by a value like c:

\footnotetext{
${ }^{*}$ Corresponding author
} 


$$
a_{1}, a_{2}=c a_{1}, a_{3}=c a_{2}, \ldots, a_{n}=c a_{n-1}, \ldots
$$

In conventional arithmetical progression, the sentences are successively added up by a value like d:

$$
a_{1}, a_{2}=a_{1}+d, a_{3}=a_{2}+d, \ldots, a_{n}=a_{n-1}+d, \ldots
$$

In this article, one of our purposes is to introduce a new progression which is a combination of arithmetical and geometrical progression called geometrical-arithmetical progression. Every sentence of this progression is a linear combination of previous sentence and is shown as:

$$
a_{1}, a_{2}=c a_{1}+d, a_{3}=c a_{2}+d, \ldots, a_{n}=c a_{n-1}+d, \ldots
$$

In the our real life, the numbers sometimes cannot be recorded or collected precisely. For example, obtained number presented as vague, can be uncertain such as "about 50" or "approximately between 100 and 110". For this reason, we try to generalize the above mentioned progression for triangular fuzzy numbers, and this is our main purpose in this article. Therefore, the fuzzy set theory attributed to Zadeh (1965) is found to be an appropriate tool in modeling the imprecise number. We prepare our discussion in 5 sections. In Section 2, we mention some of the necessary definitions and preliminaries. In Section 3, we first intruduce the Progressions of fuzzy numbers and then present a new crisp progressions. In Section 4, we first give some examples. Finally, we conclude in Section 5.

\section{Definitions and Preliminaries}

Definition1: The fuzzy subset $\tilde{N}$ of real line IR, with the membership function $\mu_{N}: I R \rightarrow[0,1]$ is a fuzzy number if and only if, (a) $\tilde{N}$ is normal, (b) $\tilde{N}$ is fuzzy convex (c) $\mu_{N}$ is upper semi-continuous, (d) supp $(\tilde{N})$ is bounded.

Definition2: A triangular fuzzy number $\tilde{N}$ is fuzzy number that membership function defined by three numbers $a_{1}<a_{2}<a_{3}$, where the base of the triangle is the interval $\left[\mathrm{a}_{1}, \mathrm{a}_{3}\right]$ and vertex is at $\mathrm{x}=\mathrm{a}_{2}$. We denote by F(IR) the set of all fuzzy number.

Definitoin3: The $\alpha$-cut of a fuzzy number $\tilde{N}$ is a non-fuzzy set defined as $\tilde{N}_{\alpha}=\left\{x \in I R ; \mu_{N}(x) \geq \alpha\right\}$. Hence we have $\tilde{N}_{\alpha}=\left[\tilde{N}_{\alpha}^{L}, \tilde{N}_{\alpha}^{U}\right]$ where

$$
\tilde{N}_{\alpha}^{L}=\inf \left\{x \in I R ; \mu_{N}(x) \geq \alpha\right\}, \tilde{N}_{\alpha}^{U}=\sup \left\{x \in I R ; \mu_{N}(x) \geq \alpha\right\}
$$

Definition4: Suppose that $\tilde{a}, \tilde{b}$ are two fuzzy numbers. Then each $\alpha$-cut $\tilde{a} \oplus \tilde{b}, \tilde{a} \Theta \tilde{b}, \tilde{a} \otimes \tilde{b}, \tilde{a} \mathrm{O} \tilde{b}$ is defined as follows:

$$
\begin{aligned}
& (\tilde{a} \oplus \tilde{b})_{\alpha}=\left[\tilde{a}_{\alpha}^{L}+\tilde{b}_{\alpha}^{L}, \tilde{a}_{\alpha}^{U}+\tilde{b}_{\alpha}^{U}\right] \\
& (\tilde{a} \otimes \tilde{b})_{\alpha}=\left[\min \left\{\tilde{a}_{\alpha}^{L} \tilde{b}_{\alpha}^{L}, \tilde{a}_{\alpha}^{L} \tilde{b}_{\alpha}^{U}, \tilde{a}_{\alpha}^{U} \tilde{b}_{\alpha}^{L}, \tilde{a}_{\alpha}^{U} \tilde{b}_{\alpha}^{U}\right\}, \max \left\{\tilde{a}_{\alpha}^{L} \tilde{b}_{\alpha}^{L}, \tilde{a}_{\alpha}^{L} \tilde{b}_{\alpha}^{U}, \tilde{a}_{\alpha}^{U} \tilde{b}_{\alpha}^{L}, \tilde{a}_{\alpha}^{U} \tilde{b}_{\alpha}^{u U}\right\}\right]
\end{aligned}
$$




$$
(\tilde{a} \mathrm{O} \tilde{b})_{\alpha}=\left(\tilde{a} \otimes\left(\frac{\tilde{1}}{b}\right)\right)_{\alpha}, \frac{\tilde{1}}{b}=\left(\frac{1}{b_{3}}, \frac{1}{b_{2}}, \frac{1}{b_{1}}\right)
$$

Definition4: $\tilde{a}$ will be crisp number with $m$ value if its membership function is

$$
\mu_{a}(x)= \begin{cases}1 & x=m \\ 0 & \text { otherwise }\end{cases}
$$

\section{Fuzzy and Crisp Progressions}

\subsection{Fuzzy Geometrical-Arithmetical Progression}

The progression $\tilde{a}_{1}, \tilde{a}_{2}, \ldots, \tilde{a}_{n}, \ldots$ is called fuzzy geometrical- arithmetical progression if we have:

$$
\tilde{a}_{1}, \tilde{a}_{2}=\tilde{1}_{\{c\}} \otimes \tilde{a}_{1} \oplus \tilde{1}_{\{d\}}, \ldots, \tilde{a}_{n}=\tilde{1}_{\{c\}} \otimes \tilde{a}_{n-1} \oplus \tilde{1}_{\{d\}}, \ldots
$$

In which $\left.\tilde{1}_{c}\right\}$ and $\tilde{1}\{d\}$ are two crisp numbers and we call them the first and second parameters of fuzzy geometrical- arithmetical progression.

Lemma1. The first and second parameters of fuzzy geometrical- arithmetical progression are in the form of:

$$
\begin{aligned}
& \tilde{1}_{\{c\}}=\left(\tilde{a}_{n} \Theta \tilde{a}_{n-1}\right) \mathrm{O}\left(\tilde{a}_{n-1} \Theta \tilde{a}_{n-2}\right) \\
& \tilde{1}_{\{d\}}=\left[\left(\tilde{a}_{n-1} \oplus \tilde{a}_{n}\right) \Theta \tilde{1}_{\{c\}} \otimes\left(\tilde{a}_{n-1} \oplus \tilde{a}_{n-2}\right)\right] \mathrm{O} \tilde{1}_{\{2\}}
\end{aligned}
$$

Proof:

$$
\tilde{a}_{n} \Theta \tilde{a}_{n-1}=\left(\tilde{1}_{\{c\}} \otimes \tilde{a}_{n-1} \oplus \tilde{1}_{\{d\}}\right) \Theta\left(\tilde{1}_{\{c\}} \otimes \tilde{a}_{n-2} \oplus \tilde{1}_{\{d\}}\right)=\tilde{1}_{\{c\}} \otimes\left(\tilde{a}_{n-1} \Theta \tilde{a}_{n-2}\right)
$$

And it implies:

$$
\tilde{1}_{\{c\}}=\left(\tilde{a}_{n} \Theta \tilde{a}_{n-1}\right) \mathrm{O}\left(\tilde{a}_{n-1} \Theta \tilde{a}_{n-2}\right)
$$

And about the second parameter, we have:

$$
\tilde{a}_{n} \oplus \tilde{a}_{n-1}=\left(\tilde{1}_{\{c\}} \otimes \tilde{a}_{n-1} \oplus \tilde{1}_{\{d\}}\right) \oplus\left(\tilde{1}_{\{c\}} \otimes \tilde{a}_{n-2} \oplus \tilde{1}_{\{d\}}\right)=\tilde{1}_{\{c\}} \otimes\left(\tilde{a}_{n-1} \oplus \tilde{a}_{n-2}\right) \oplus \tilde{1}_{\{2\}} \otimes \tilde{1}_{\{d\}}
$$

And it implies:

$$
\tilde{1}_{\{d\}}=\left[\left(\tilde{a}_{n-1} \oplus \tilde{a}_{n}\right) \Theta \tilde{1}_{\{c\}} \otimes\left(\tilde{a}_{n-1} \oplus \tilde{a}_{n-2}\right)\right] \mathrm{O} \tilde{1}_{\{2\}}
$$

Lemma2. The $n^{\text {th }}$ sentence of fuzzy geometrical- arithmetical progression is in the form of: 


$$
\tilde{a}_{n}=\tilde{1}_{\left\{c^{n-1}\right\}} \otimes \tilde{a}_{1} \oplus \tilde{1}_{\{d\}} \otimes \bigoplus_{k=2}^{n} \tilde{1}_{\left\{c^{n-k}\right\}}
$$

Proof:

$$
\begin{aligned}
& \tilde{a}_{2}=\tilde{1}_{\{c\}} \otimes \tilde{a}_{1} \oplus \tilde{1}_{\{d\}} \\
& \tilde{a}_{3}=\tilde{1}_{\{c\}} \otimes \tilde{a}_{2} \oplus \tilde{1}_{\{d\}}=\tilde{1}_{\left\{c^{2}\right\}} \otimes \tilde{a}_{1} \oplus \tilde{1}_{\{c\}} \otimes \tilde{1}_{\{d\}} \oplus \tilde{1}_{\{d\}} \\
& \tilde{a}_{4}=\tilde{1}_{\{c\}} \otimes \tilde{a}_{3} \oplus \tilde{1}_{\{d\}}=\tilde{1}_{\left\{c^{3}\right\}} \otimes \tilde{a}_{1} \oplus \tilde{1}_{\left\{c^{2}\right\}} \otimes \tilde{1}_{\{d\}} \oplus \tilde{1}_{\{c\}} \otimes \tilde{1}_{\{d\}} \oplus \tilde{1}_{\{d\}} \\
& \quad \cdot \\
& \quad \cdot \\
& \tilde{a}_{n}=\tilde{1}_{\{c\}} \otimes \tilde{a}_{n-1} \oplus \tilde{1}_{\{d\}}=\tilde{1}_{\left\{c^{n-1}\right\}} \otimes \tilde{a}_{1} \oplus \tilde{1}_{\left\{c^{n-2}\right\}} \otimes \tilde{1}_{\{d\}} \oplus \ldots \oplus \tilde{1}_{\{d\}}=\tilde{1}_{\left\{c^{n-1}\right\}} \otimes \tilde{a}_{1} \oplus \tilde{1}_{\{d\}} \otimes \bigoplus_{k=2}^{n} \tilde{1}_{\left\{c^{n-k}\right\}}
\end{aligned}
$$

Lemma3. The sum of the first $n$ terms of geometrical- arithmetical progression is in the form of:

Proof:

$$
\left.\left.\left.\tilde{S}_{n}=\tilde{a}_{1} \otimes \underset{k=0}{n-1} \tilde{1}_{\{c} k\right\} \oplus \tilde{1}_{d}\right\} \underset{k=0}{\oplus-2} \tilde{1}_{\{n-k-1\}} \otimes \tilde{1}_{\{c} k\right\}
$$

$$
\begin{aligned}
\tilde{S}_{n}= & \tilde{a}_{1} \oplus \tilde{a}_{2} \oplus \ldots \oplus \tilde{a}_{n}=\tilde{a}_{1} \oplus\left(\tilde{1}_{\{c\}} \otimes \tilde{a}_{1} \oplus \tilde{1}_{\{d\}}\right) \oplus \\
& \left(\tilde{1}_{\left\{c^{2}\right\}} \otimes \tilde{a}_{1} \oplus \tilde{1}_{\{c\}} \otimes \tilde{1}_{\{d\}} \oplus \tilde{1}_{\{d\}}\right) \oplus \ldots \oplus\left(\tilde{1}_{\left\{c^{n-1}\right\}} \otimes \tilde{a}_{1} \oplus \tilde{1}_{\left\{c^{n-2}\right\}} \otimes \tilde{1}_{\{d\}} \oplus \ldots \oplus \tilde{1}_{\{d\}}\right) \\
= & \left(\tilde{1}_{\{c\}} \oplus \ldots \oplus \tilde{1}_{\left\{c^{n-1}\right\}}\right) \otimes \tilde{a}_{1} \oplus \tilde{1}_{\{n-1\}} \otimes \tilde{1}_{\{d\}} \oplus \tilde{1}_{\{n-2\}} \otimes \tilde{1}_{\{c\}} \otimes \tilde{1}_{\{d\}} \oplus \ldots \oplus \tilde{1}_{\{n-(n-1)\}} \otimes \tilde{1}_{\left\{c^{n-2}\right\}} \otimes \tilde{1}_{\{d\}} \\
= & \tilde{a}_{1} \otimes \bigoplus_{k=0}^{n-1} \tilde{1}_{\left\{c^{k}\right\}} \oplus \tilde{1}_{\{d\}} \otimes \bigoplus_{k=0}^{n-2} \tilde{1}_{\{n-k-1\}} \otimes \tilde{1}_{\left\{c^{k}\right\}}
\end{aligned}
$$

\subsection{Fuzzy Geometrical Progression}

Through substituting $d=0$ in the fuzzy geometrical-arithmetical progression, there will be a geometrical progression of fuzzy numbers out of which:

$$
\begin{aligned}
& \tilde{1}_{\{c\}}=\left(\tilde{a}_{n} \Theta \tilde{a}_{n-1}\right) \mathrm{O}\left(\tilde{a}_{n-1} \Theta a_{n-2}\right) \\
& \tilde{a}_{n}=\tilde{1}\left\{c^{n-1}\right\} \otimes \tilde{a}_{1} \\
& \tilde{S}_{n}=\tilde{a}_{1} \otimes \bigoplus_{k=0}^{n-1} \tilde{l}_{\left\{c^{k}\right\}}
\end{aligned}
$$

Remark 1: A fuzzy geometric series is defined the sum of the terms in a fuzzy geometric progression.

\subsection{Fuzzy Arithmetical Progression}


Through substituting $c=1$ in the fuzzy geometrical- arithmetical progression, there will be an arithmetical progression of fuzzy numbers out of which:

$$
\begin{aligned}
& \tilde{1}_{\{d\}}=\left(\tilde{a}_{n} \Theta \tilde{a}_{n-2}\right) \otimes \tilde{1}_{\{2\}} \\
& \tilde{a}_{n}=\tilde{a}_{1} \oplus \tilde{1}_{\{n-1\}} \otimes \tilde{1}_{\{d\}} \\
& \tilde{S}_{n}=\tilde{1}_{\{n\}} \otimes \tilde{a}_{1} \oplus \tilde{1}_{\left\{\frac{n(n-1)}{2}\right\}} \otimes \tilde{1}_{\{d\}}
\end{aligned}
$$

Remark 2: A fuzzy arithmetic series is defined the sum of the terms in a fuzzy arithmetic progression.

\subsection{Geometrical-Arithmetical Progression of crisp numbers}

If the fuzzy numbers of $\tilde{a}_{1}, \tilde{a}_{2}, \ldots, \tilde{a}_{n}, \ldots$ change into the crisp numbers $a_{1}, a_{2}, \ldots, a_{n}, \ldots$, then there will be a progression called the geometrical- arithmetical progression of crisp numbers as follows:

$$
a_{1}, a_{2}=c a_{1}+d, \ldots, a_{n}=c a_{n-1}+d, \ldots
$$

Considering the previous lemmas, we have

$$
\begin{aligned}
& c=\frac{a_{n}-a_{n-1}}{a_{n-1}-a_{n-2}} \quad, \quad d=\frac{\left(a_{n-1}+a_{n}\right)-c\left(a_{n-1}+a_{n-2}\right)}{2} \\
& a_{n}=c^{n-1} a_{1}+d \sum_{k=2}^{n} c^{n-k} \\
& S_{n}=a_{1} \sum_{k=0}^{n-1} c^{k}+d \sum_{k=0}^{n-2}(n-k-1) c^{k}
\end{aligned}
$$

Remark 3: If the value 1 is placed instead of $c$ in the geometrical- arithmetical progression, then this progression will change in to an arithmetical progression.

Remark 4: If the value 0 is placed instead of $d$ in the geometrical- arithmetical progression, then this progression will change in to a geometrical progression.

\section{Numerical Examples}

Example 1: suppose we have the geometrical- arithmetical progression $4,13,40, \ldots$. Then there will be:

$$
c=3, d=1, S_{5}=542
$$

Example 2: suppose we have the fuzzy geometrical- arithmetical progression $\tilde{3}, \tilde{9}, \tilde{2} 1, \ldots$ out of which:

$$
\tilde{3}=(2,3,4), \tilde{9}=(7,9,11) \quad, \tilde{2} 1=(17,19,25)
$$

then

$$
\tilde{1}_{\{c\}}=(\tilde{2} 1 \Theta \tilde{9}) \mathrm{O}(\tilde{9} \Theta \tilde{3})=(10,12,14) \mathrm{O}(5,6,7)=(2,2,2)
$$




$$
\begin{aligned}
& \tilde{1}_{\{d\}}=((\tilde{2} 1 \oplus \tilde{9}) \Theta(2,2,2)(\tilde{9} \oplus \tilde{3})) \mathrm{O}(2,2,2)=(3,3,3) \\
& \tilde{S}_{10}=(140,171,202) \\
& \tilde{S}_{10, \alpha}=[140+31 \alpha, 202-31 \alpha]
\end{aligned}
$$

Figure 1 shows sum of the first $n$ terms in Example 2.

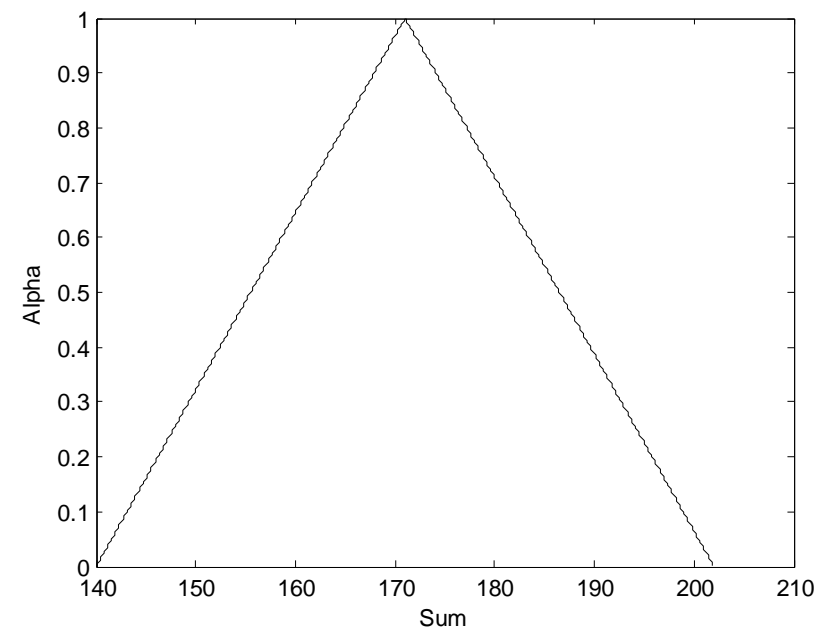

Figure 1. Fuzzy number of the sum of the first $n$ terms

Example 3: A population increase 5\% each year on the basis of birth rate considering that it admits 10 peopole as immiggrants. According to the obtained information, the primary population is approximately 200 people. On the whole, the relation between the population in $n^{\text {th }}$ year and and $r$ brith rate, and admitting to $d$ number follows:

$$
\begin{aligned}
& \tilde{a}_{0}=(195,200,205) \\
& \tilde{a}_{n}=\tilde{1}_{\left\{(1+r)^{n}\right\}} \otimes \tilde{a}_{0} \oplus \bigoplus_{k=1}^{n} \tilde{1}_{\{d\}} \otimes \tilde{1}_{\left\{(1+r)^{n-k}\right\}}
\end{aligned}
$$

In this example $r=0.05, d=10$

$$
\begin{aligned}
& a_{n}=\tilde{1}_{\left\{(1.05)^{n}\right\}} \otimes(195,200,205) \oplus \bigoplus_{k=1}^{n} \tilde{1}_{\{10\}} \otimes \tilde{1}_{\left\{(1.05)^{n-k}\right\}} \\
& =\left(195(1.05)^{n}+10 \sum_{k=1}^{n}(1.05)^{n-k}, 200(1.05)^{n}+10 \sum_{k=1}^{n}(1.05)^{n-k}, 205(1.05)^{n}+10 \sum_{k=1}^{n}(1.05)^{n-k}\right)
\end{aligned}
$$

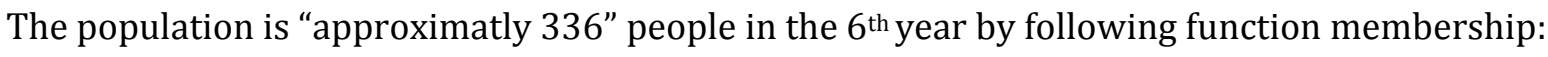

$$
\begin{aligned}
& \tilde{a}_{6}=(329.32,336.02,342.72) \\
& \tilde{a}_{6, \alpha}=[329.32+6.7 \alpha, 342.72-6.7 \alpha]
\end{aligned}
$$

Figure 2 shows number of people in the $6^{\text {th }}$ year in Example 3. 


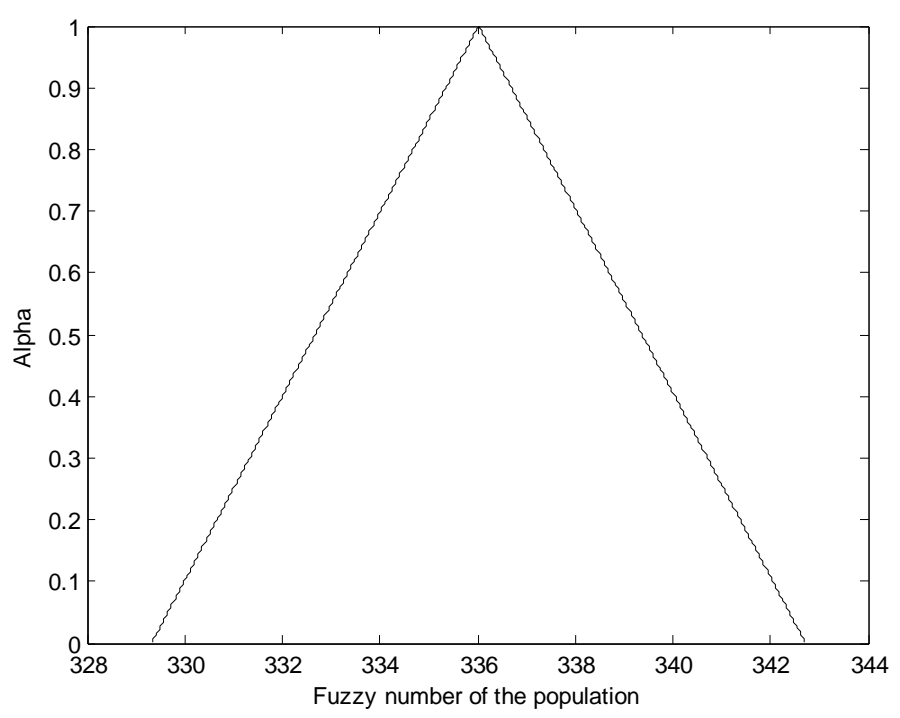

Figure 2. Fuzzy number of the population

\section{Conclusion}

In traditional method, the number sequences is generally assumed to be a crisp value. However, real numbers are usually vague, so conventional formulas of progressions are inaccurate. The application of fuzzy sets theory to progressions is proposed in this paper by triangular fuzzy number. We have introduced the fuzzy progression and a new crisp progression. We have prepared the computational procedure to find some properties of the progressions of fuzzy numbers. Our methods are well defined since if all the numbers are crisp they reduce traditional method.

\section{References}

[1] Abramowitz, M., Stegun, I. A. (Eds.), "Handbook of Mathematical Functions with Formulas, Graphs, and Mathematical Tables", 9th printing. New York: Dover, pp. 10, 1972.

[2] Dubois, D., and Prade, H., "Operations of fuzzy numbers", International Journal of Systems Science, 9, pp. 613-626, 1978.

[3] Dubois, D., H. Prade, "Operations on fuzzy numbers", International Journal of Systems Sciences, 9, pp.613-626, 1978.

[3] E. Pasha, A. Saiedifar and B. Asady, "The percentiles of fuzzy numbers and their applications", Iranian Journal of Fuzzy Systems, 6(1), pp. 27-44, 2009.

[4] Zadeh, L. (1965), "Fuzzy Sets", Information Control, 8, pp. 338-353, 1965. 